\title{
Effects of Wall Perturbations in Multimode Waveguides
}

\author{
S. W. Maley and E. Bahar
}

\author{
Contribution From Electrical Engineering Department, University of Colorado, Boulder, Colo.
}

\begin{abstract}
An experimental investigation has been made of the effects of wall perturbations in multimode rectangular waveguides, whose dimensions were such that the modes $\mathrm{TE}_{n o}$ for $n=1,2$. . 15 were propagating modes and all of the others were cutoff. Measurements were made at $X$-band frequencies. The investigation has shown that when the lowest ordered mode is incident upon a perturbation in the waveguide wall, all of the higher ordered propagating modes will be excited in both directions in the waveguide, but the distribution of energy among the modes is critically dependent upon the shape and size of the perturbation.

Measurements on this waveguide with various wall perturbations may be used to gain insight into the effects of ionospheric perturbations on terrestrial VLF propagation.
\end{abstract}

\section{Introduction}

Perturbations in the walls of waveguides can in the general case excite all orders of modes traveling away from the perturbation. In the case of a waveguide propagating only a single mode, the higher modes are of little importance except in the immediate vicinity of the perturbation. However, in a waveguide which propagates more than one mode, several of the higher modes excited by a perturbation can be significant at great distances from it. In such a waveguide the relative amplitudes and phases of the various modes are dependent upon the shape and size of the perturbation. Because of this dependence it is possible, in theory at least, to gain information about the size and shape of a perturbation from measurements of the various propagating modes. This principle might therefore be used to gain information about perturbations of the ionosphere from measurements of VLF propagation in the earth-ionosphere waveguide. It is felt that some insight into the effects of ionospheric perturbations upon VLF propagation can be acquired by the laboratory study of the effects of wall perturbations in a microwave waveguide having a width, in wavelengths at the microwave frequency of operation, equal to the height of the earth-ionosphere waveguide in wavelengths at the VLF frequency of interest. The boundaries of the microwave waveguide should be chosen so that their reflection coefficients are the same as those in the earth-ionosphere waveguide. It is theoretically possible to select the materials and dimensions of the microwave waveguide so that it is an exact scale model of the earthionosphere waveguide; then the electromagnetic behavior of the model will precisely give the electromagnetic behavior of the earth-ionosphere waveguide. The technical problems involved in the construction of an exact scale model are formidable; therefore it was decided that the initial investiga-

1 This work was supported by the Advanced Research Projects Agency A RPA Order No. 183-62, NBS Contract CST-7348. tions would be made with a simple waveguide of rectangular cross section. Such a waveguide bears little resemblance to the earth-ionosphere waveguide; it transmits power along an axis rather than between two spheres as in the earth-ionosphere waveguide. However, if its width is properly chosen and the proper wall materials are chosen, then its behavior for certain wall perturbations should be similar to that of the earth-ionosphere waveguide. J. R. Wait has recently given strong theoretical justification for this sort of experimental study [Wait, 1963]. A further important use of such a waveguide is that of checking the accuracy of various approximate theoretical solutions for fields in waveguide having wall perturbations [Wait and Murphy, 1957; Wait, 1960a, 1960b, 1961, 1962; Morgan, 1950].

\section{Theory}

The earth can usually be considered as a good conductor at VLF, therefore it is a straightforward matter to represent it in an electromagnetic model. It is simply necessary to use a material having a conductivity equal to that of the earth multiplied by an appropriate scale factor. Furthermore, since the conductivity is scaled upward when the size is scaled downward it follows that, in a model small enough to be placed in a laboratory, the conductivity of the material representing the earth would be very high, so high, in fact, that a perfect conductor can be used with very little error.

On the other hand, the representation of the onosphere in the model is somewhat more difficult. The magnitude of the refractive index of the ionosphere at VLF under certain rather common conditions is approximately unity; it can range somewhat above or below this figure. It is more difficult to model a medium with a refractive index $<1$ than one with a refractive index $>1$. Therefore it was decided to first model the case for which the refractive 
index, $N$, is defined by ${ }^{2}$

$$
N^{2}=1-\frac{i}{L}
$$

where $L$ is a positive number ranging from about 0.1 to 3 . The surface impedance, $Z$, corresponding to this refractive index is

$$
Z=\frac{\eta_{0}}{1-\frac{i}{L}}\left(\cos ^{2} \theta-\frac{i}{L}\right)^{1 / 2}
$$

where

$$
\eta_{0} \cong 120 \pi
$$

and $\theta$ is the angle of incidence. For the lower ordered modes, the angle of incidence is close to $\frac{\pi}{2}$; and, for such angles the surface impedance, as given above, has an imaginary part which is negative for large $L$ and positive for small $L$. A suitable model, therefore, would be as shown in figure 1. The bottom wall would be a very good conductor, and the top wall would be a material having a surface impedance given by the above equation for $Z$. The desired modes of operation of the waveguide in figure 1 are TM modes which have no tangential components of the magnetic field intensity, $\bar{H}$, on the side walls and no variation of the field quantities in the direction of the normal to the broad walls. This would require that the broad walls have an essentially infinite permeability. Such walls are difficult to even approximate; therefore it was decided that the type of wave guide shown in figure 1 is unsuitable. J. R. Wait has suggested a way to

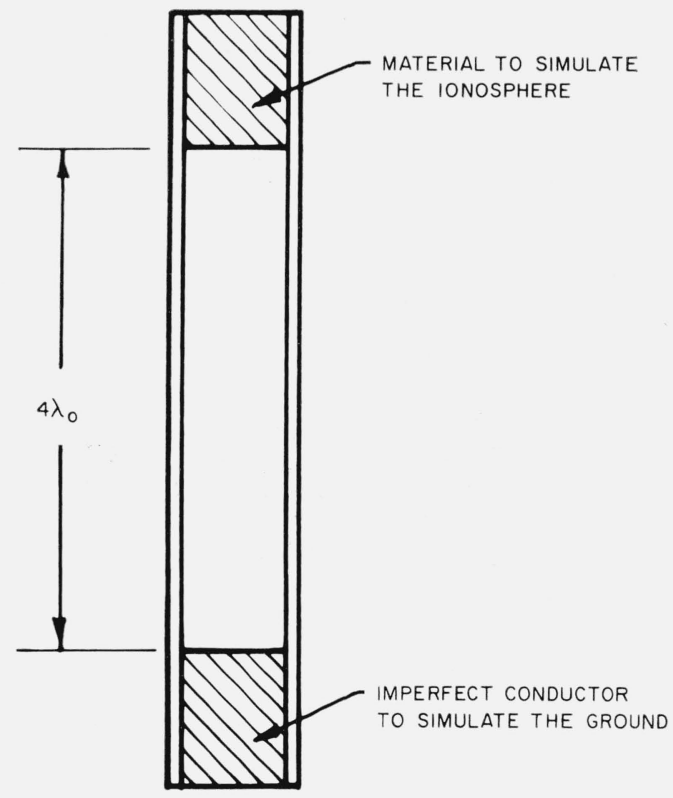

Figure 1. Cross section of a possible laboratory waveguide, a two-dimensional model of the earth-ionosphere region.

2 The rationalized M.K.S. system of units will be used. The time dependence is assumed to be $e^{i \omega t}$, where $i=\sqrt{-1}, \omega$ is angular frequency and $t$ is time. overcome the disadvantages of the waveguide of figure 1 [Maley and Bahar, 1963]. He suggested the one shown in figure 2 . The part of the waveguide above the plane of symmetry in figure 2 may be considered as an electromagnetic dual of the waveguide in figure 1 for the case in which the bottom wall in figure 1 is a perfect conductor. The term electromagnetic dual is used to mean that the fields and the parameters are interchanged according to the relations

$$
\begin{aligned}
& \bar{H} \rightarrow \bar{E}, \\
& \bar{E} \rightarrow-\overline{\mathrm{H}}, \\
& \mu \rightarrow \epsilon, \\
& \epsilon \rightarrow \mu,
\end{aligned}
$$

where, in general, $\epsilon$ and $\mu$ are complex. Maxwell's equations are invariant to this transformation; so fields which exist in an electromagnetic system can be directly related to those which exist in the dual of the system. Thus if the field is measured in the waveguide of figure 2 , the field in the waveguide of figure 1 can be calculated from the measurements by the above transformation. Using this transformation, the bottom wall in figure 1 (this wall is assumed to be a perfect conductor) transforms into a plane along which there can be no tangential component of $\bar{H}$. But this is exactly the behavior of the plane of symmetry of figure 2 when only ${ }^{\mathrm{TE}_{n 0}}$ modes having $\bar{E}$ symmetric about the plane of symmetry are considered. In a completely symmetric system only these modes exist, and therefore the plane of

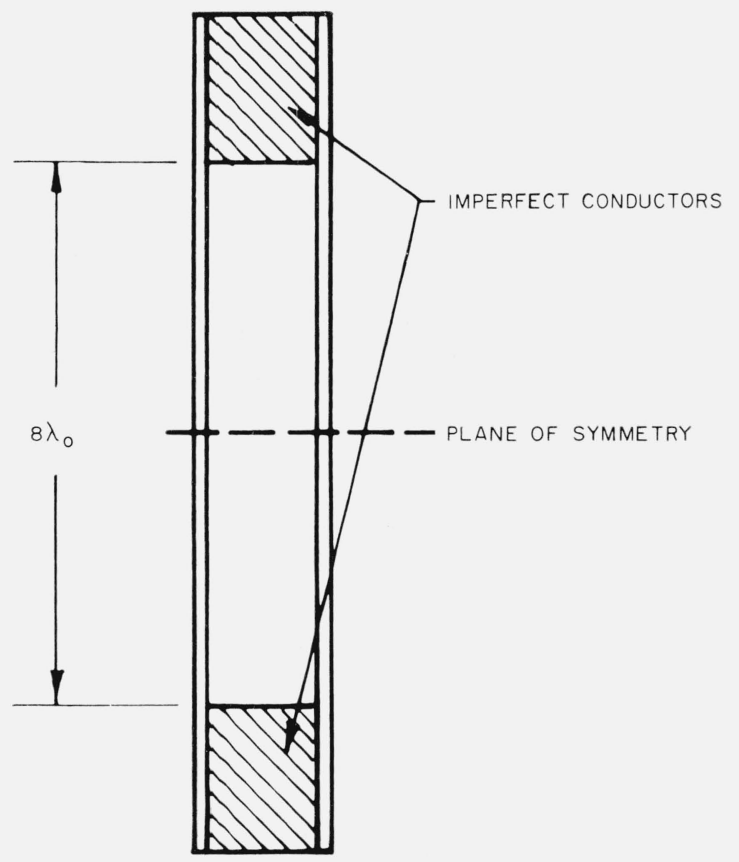

Figure 2. Cross section of the laboratory waveguide used in the experimental investigation. 
symmetry may be regarded as the exact dual of a perfect conductor. The top wall in figure 1 transforms into a wall having a surface admittance, $Y$, equal to

$$
Z\left(\eta_{0}\right)^{-2}=\frac{\left(\cos ^{2} \theta-i L\right)^{1 / 2}}{\eta_{0}(1-i L)}
$$

The broad walls of figure 1 are transformed into perfect conductors. The waveguide in figure 2 can be approximated to a good order of accuracy using readily available materials. The broad walls can be made of any metallic substance and the top and bottom walls can be made of commercially available imperfectly conducting materials. Since it is desired that there be no variations of the field quantities in the horizontal direction in figure 2 , it is advisable to make the narrow dimension of the waveguide sufficiently small that only $\mathrm{TE}_{n 0}$ modes can propagate. For the lower ordered modes in the earth ionosphere waveguide, the reflection coefficient of the ionosphere, $R_{i}$, given by [Wait, 1960a]

$$
R_{i}=\frac{\cos \theta-Z \eta_{0}^{-1}}{\cos \theta+Z \eta_{0}^{-1}}
$$

can be roughly approximated by -1 which in the dual model corresponds to +1 or a perfect conductor. Therefore the first investigations were made with brass top and bottom walls on the waveguide. Later an imperfectly conducting material was used to give a better approximation; a still closer approximation is now being planned.

\section{Experimental Equipment}

The model waveguide was constructed from three 8-ft long sections with uniform cross section 25.4 $\mathrm{cm} \times 0.95 \mathrm{~cm}$. The operating frequency was $9 \cdot 10^{9} \mathrm{c} / \mathrm{s}$, hence the length of the waveguide was about 225 $\lambda_{0}$ ( $\lambda_{0}$ being the free space wavelength) representing $4,500 \mathrm{~km}$ in the earth-ionosphere waveguide at 15 $\mathrm{kc} / \mathrm{s}$. The width of the waveguide was slightly over $7.5 \lambda_{0}$ and the height was less than half a wavelength. Symmetry was maintained in the waveguide at all times (even when perturbations were inserted) to maintain the dual boundary conditions, hence only the first eight $\mathrm{TE}_{n, 0}$ modes with symmetric electric fields (having $n$ only odd integers) were propagating modes, the others being evanescent. A nonreflecting load was used to terminate the waveguide. The "perfectly conducting" narrow walls of the waveguide represent the idealized ionospheric boundary in the earth-ionosphere waveguide (having a vanishing tangential magnetic field). To simulate the physical characteristics of the ionosphere more closely, these walls will be padded with a lossy dielectric material that is now in the process of being manufactured.

At large distances from a transmitting vertical dipole in the earth-ionosphere waveguide the field would be represented essentially by a $\mathrm{TM}_{1,0}$ mode, being the least attenuated of all the propagating modes. Hence it was desired to launch a $\mathrm{TE}_{1,0}$ mode into the model waveguide. This was obtained by transmitting a $\mathrm{TE}_{1,0}$ mode from a standard $\dot{X}$-band waveguide through a $2.5 \mathrm{~m}$ long taper with a sinusoidal profile into the model waveguide. The wave thus launched into the model waveguide had a higher mode content (essentially $\mathrm{TE}_{3,0}$ ) of 8 percent approximately.

Amplitude and phase measurements were carried out by probing the electric field along the center line of the waveguide and across transverse sections of it, through a series of holes, rather than slots. In order to observe the detail interference structure of the field pattern it was necessary to space the holes such that at least eight readings would be taken per free space wavelength along the length of the waveguide. It was necessary to take a minimum of 500 readings on either side of the perturbed region to recognize the presence of all the higher modes propagating in the waveguide.

A block diagram of the amplitude and phase measuring system is given in figure 3 . Since extensive amplitude data was to be obtained, it was desired to have this quantity plotted directly. The amplitude modulated signal sampled by a probe with a square law detector was amplified and rectified. This rectified signal was passed through a square root circuit before being recorded on an $X-Y$ recorder. The $X$ axis representing the position of the probe and the $Y$ axis the amplitude of the electric field at the sampling holes. Phase readings were taken by comparing the phase of the sampled electric field with that of a reference signal in a Magic Tee with the help of a precision phase shifter. A general view of the system with only one section connected to the taper section is shown in figure 4.

\section{Electric Field Measurements}

The first series of experiments were conducted with the uniform cross section of the waveguide locally perturbed by sinusoidal shaped slabs (of the same material as the waveguide walls) placed symmetrically along the walls. The first pair of perturbations were approximately $2 \frac{1}{2} \quad \lambda_{0}$ long such that the half width of the waveguide varied as

$$
h(z)=h\left[1-a \cos \frac{\pi\left(z-z_{0}\right)}{2 z_{0}}\right], \quad z_{0} \geqq z \geqq-z_{0} ;
$$

$h$ is the half width of the uniform waveguide and $(h a)$ is the maximum height of the perturbation and $2 z_{0}$ is the length of the perturbation (see fig. 5), $a=0.2$ representing a 20 percent local depression in the height of the ionosphere.

Measurements of the transverse and longitudinal variations of the electric field (amplitude and phase) were taken on both sides of the perturbation. The amplitude measurement along the axis of the guide revealed distinctly different standing wave patterns on either side of the perturbation (fig. 6a). These patterns could be precisely explained theoretically in terms of interaction between the principal mode 
Coaxial Cable

$\longrightarrow$ Weference Signal Path

Matched

Termination

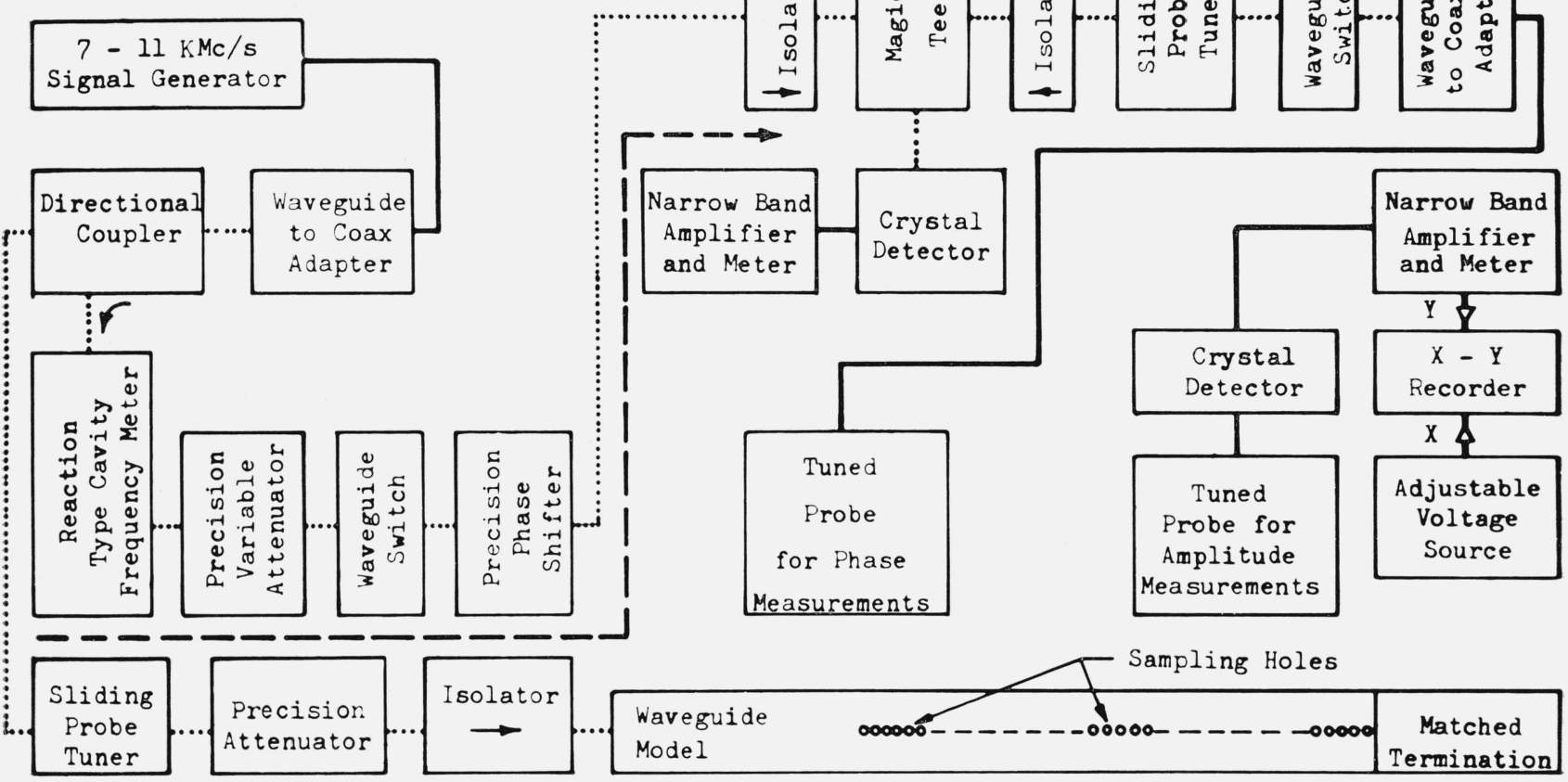

FIGURE 3. Block diagram of the system used for measurement of the electric field intensity and phase

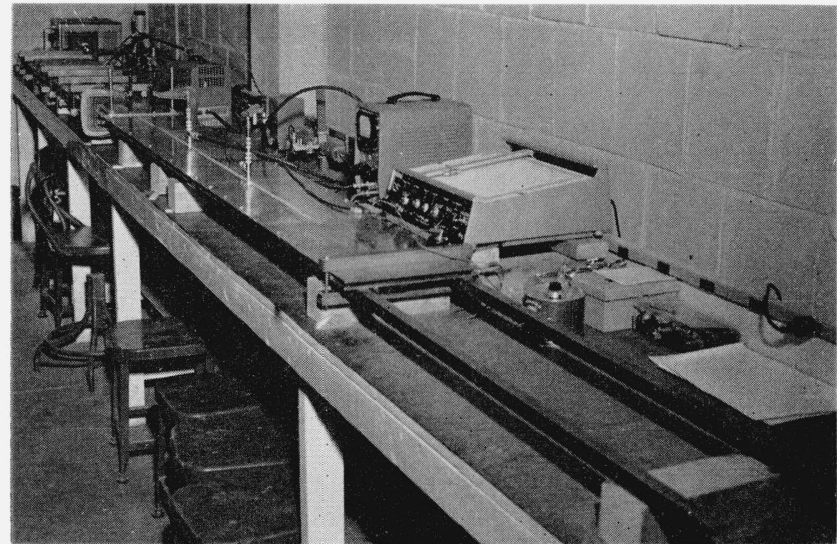

Figure 4. General view of a section of the model waveguide backed by the amplitude and phase measuring device.

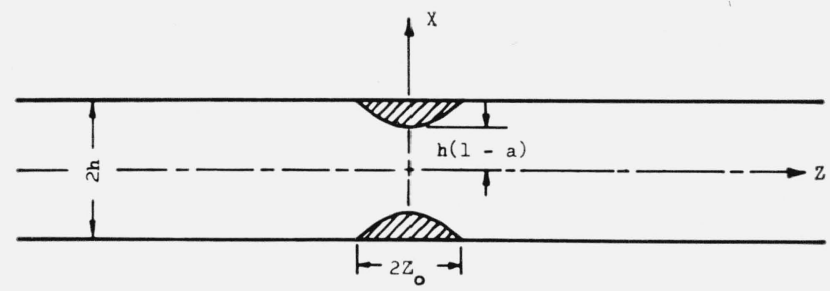

Figure 5. Axial section of waveguide with sinusoidal perturbation inserted. 

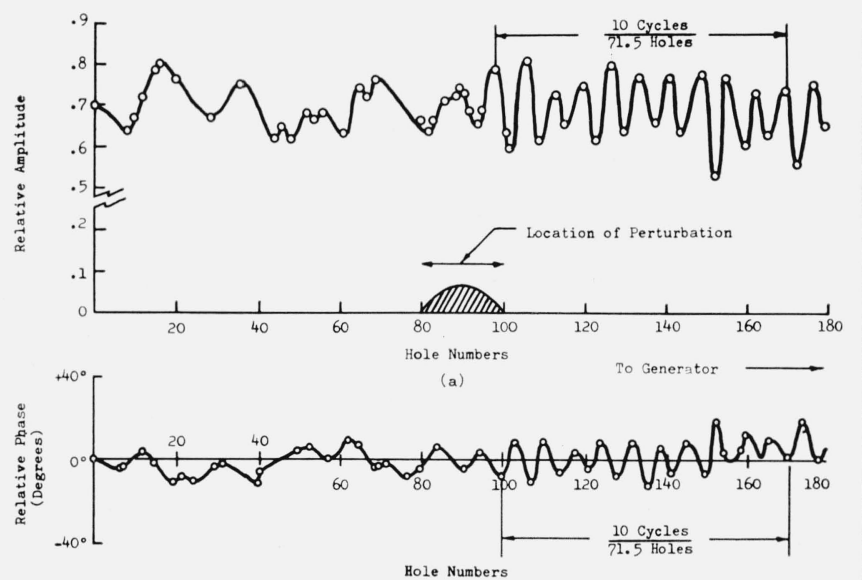

(b)

Figure 6, a, b. Axial amplitude and phase variations with sinusoidal perturbation $\mathrm{a}=0.2$.

and the higher order modes generated by the perturbation. While the phase variation along the axis of the waveguide was very close to linear, the phase anomaly $\Delta \varphi$ (difference between the phase variation along the axis of the waveguide in the perturbed case and in the unperturbed case) remarkably resembled the amplitude variations (fig. $6 \mathrm{~b})$. This too can be accounted for in terms of mode interaction. The transverse amplitude variation (fig. 7a) emphasized the higher mode content. The very near symmetry of the measured variations implied that only the odd modes were being excited as is required by the duality relationship between the model and the actual problem. The transverse phase variations (fig. 7b) also clearly showed up the higher mode content.

To explain the close correspondence between the axial amplitude and phase anomaly variations in the perturbed waveguide, one notes, for instance, that

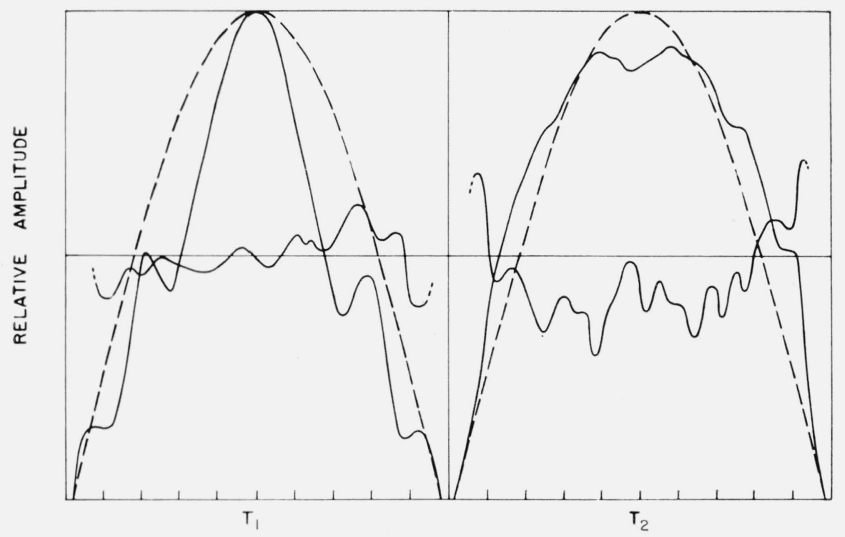

Figure 7, a, b. Transverse amplitude and phase variations with sinusoidal perturbation $\mathrm{a}=0.2$.

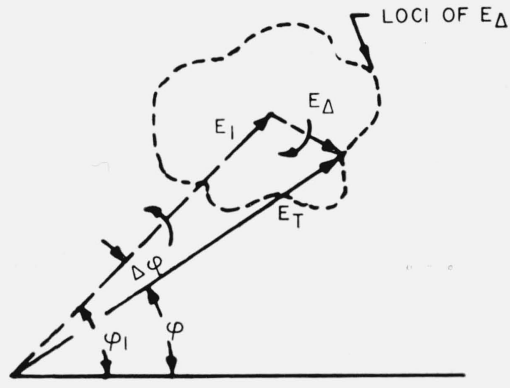

Figure 8a. Total electric field represented by higher modes added to principal mode.

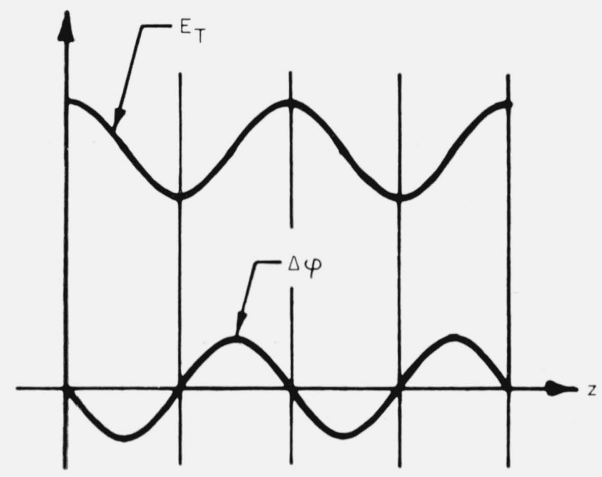

Figure 8b. Relationship between amplitude and phase anomaly.

in the region between the generator and the perturbation the resultant field comprises the (forward propagating) principal mode and a sum of reflected (backward propagating) modes.

In the schematic representation of the resultant field variations along the axis of the waveguide (fig. 8a) $E_{1}$ and $\varphi_{1}$ are the unperturbed amplitude and phase of the electric field (principal mode). $E_{t}$ and $\varphi$ are the resultant (perturbed) amplitude and phase of the electric field, while $E_{\Delta}$ and $\Delta \varphi$ are the amplitude and phase anomaly. Note that while $E_{1}$ rotates counterclockwise, $E_{\Delta}$ rotates clockwise (towards generator). Hence, while the standing wave patterns of $E_{t}$ and $\Delta \varphi$ have the same wavelength, and the variation of $E_{t}$ lags that of $\Delta \varphi$ by $\pi / 2$ radians (fig. 8 b).

While the standing wave pattern in a one mode system (in which only one mode propagating in each direction is present) has a standing wave wavelength of $1 / 2 \lambda_{g}$, those measured in the perturbed (multimode) waveguide were $0.85 \lambda_{g}$ in the region between she generator and the perturbation and about $1.2 \lambda_{g}$ in the region beyond the perturbation 
The total field in the region between the generator and the perturbation is approximately

$$
\begin{aligned}
& E_{t}=E_{1}^{\mathrm{I}}\left\{1+R_{1}^{\mathrm{I}} e^{i 2 \pi}\left(\frac{1}{\lambda_{g 1}}+\frac{1}{\lambda_{g 1}}\right) z\right. \\
& +\ldots+R_{n}^{\mathrm{I}} e^{i 2 n}\left(\frac{1}{\lambda_{g 1}}+\frac{1}{\lambda_{g n}}\right) z+\ldots \\
& \left.+R_{15}^{\mathrm{I}} e^{i 2 \pi}\left(\frac{1}{\lambda_{g 1}}+\frac{1}{\lambda_{g 15}}\right) z\right\} \\
& n=1,3,5 . . .15, \quad-\left(z-z_{0}\right)>\lambda_{0} ;
\end{aligned}
$$

and in the region beyond the perturbation

$$
\begin{gathered}
E_{\mathrm{II}}=E_{1}^{1}\left\{R_{\mathrm{I}}^{1 \mathrm{I}}+R_{3}^{\mathrm{II}} e^{i 2 \pi}\left(\frac{1}{\lambda_{g 1}}-\frac{1}{\lambda_{g 3}}\right) z\right. \\
+\ldots R_{n}^{\mathrm{II}} e^{i 2 \pi}\left(\frac{1}{\lambda_{g n}}-\frac{1}{\lambda_{g 1}}\right) z+\ldots \\
\left.+R_{15}^{\mathrm{II}} e^{i 2 \pi}\left(\frac{1}{\lambda_{g 1}}-\frac{1}{\lambda_{g 15}}\right) z\right\} \\
\quad n=1,3,5,7 . .15, \quad\left(z-z_{0}\right)>\lambda_{0},
\end{gathered}
$$

where $E_{1}^{\mathrm{I}}$ is the incident principal (forward propagating) mode in region $\mathrm{I}$ and $E_{1}^{\mathrm{I}} R_{1}^{\mathrm{II}}=E_{1}^{\mathrm{II}}$ is the principal mode transmitted into region II. $R_{n}^{\mathrm{I}}$ $=E_{n}^{\mathrm{I}} / E_{1}^{\mathrm{I}}$ and $R_{n}^{\mathrm{II}}=E_{n}^{\mathrm{II}} / E_{1}^{\mathrm{I}}$ are the reflected and transmitted higher mode conversion coefficients respectively, $z$ is the distance along the axis of the waveguide and $\lambda_{g n}$ the waveguide wavelength of the $n$th mode.

The interaction between the 15th mode (backward propagating) and the principal mode in region I has an effective standing-wave wavelength, $\lambda_{\text {eff } 15}^{I}$;

$$
\frac{1}{\lambda_{\text {eff } 15}^{I}}=\frac{1}{\lambda_{g 1}}+\frac{1}{\lambda_{g 15}} ; \lambda_{\text {eff } 15}^{I}=\frac{\lambda_{g 1} \lambda_{g 15}}{\lambda_{g 1}+\lambda_{g 15}}
$$

Now $\lambda_{g n}=\lambda_{0}\left[1-\left(\frac{n \lambda_{0}}{4 h}\right)^{2}\right]^{-1 / 2}=\frac{\lambda_{0}}{\cos \theta_{n}}$; where the free space wavelength $\lambda_{0}=3.33 \mathrm{~cm}$ and the half width of the waveguide $h=12.7 \mathrm{~cm}$. Thus

$$
\lambda_{\text {eff } 15}^{I}=\lambda_{g 1} \frac{\cos \theta_{1}}{\cos \theta_{1}+\cos \theta_{n}}=0.85 \lambda_{g 1} .
$$

Similarly, in region I beyond the perturbation, the interaction between the 15th (forward propagating) mode and the principal mode has an effective standing-wave wavelength

$$
\lambda_{\text {eff }}^{\mathrm{II}}=\frac{\lambda_{g 1} \lambda_{g 15}}{\lambda_{g 15}-\lambda_{g 1}}=\lambda_{g 1} \frac{\cos \theta_{1}}{\cos \theta_{1}-\cos \theta_{15}}=1.22 \lambda_{g 1} .
$$

From the above results it seems that for the particular type of perturbation introduced into the waveguide in the above experiment $R_{15}$ was the largest of all the mode conversion coefficients, since a predominant $\lambda_{\text {eff 15 }}^{\mathrm{I}}=0.85 \lambda_{g 1}$ and $\lambda_{\text {eff } 15}^{\mathrm{II}}=1.22 \lambda_{g 1}$ were actually obtained from the amplitude and phase data taken. Looking into the transverse field distributions of the different modes one could perhaps draw some qualitative explanation for the observation that $R_{15}$ is the largest of all the mode conversion coefficients for the particular form of perturbation used in the above experiment.

Figure 9 is the pattern of the electric field distribution across the waveguide for the $\mathrm{TE}_{15,0}$ mode in the uniform cross section $(0.95 \mathrm{~cm} \times 25.4 \mathrm{~cm})$ waveguide. The first null in the electric field is $\frac{2 h}{n}=\frac{25.4}{15}=1.7 \mathrm{~cm}$ from the uniform waveguide wall. In general, for the $n$th mode the first null will be $\frac{25.4}{n} \mathrm{~cm}$ from the waveguide wall.

The average height of the sinusoidal perturbation used in the above experiments is $\frac{2}{\pi} \times 2.54 \mathrm{~cm}=1.62$ $\mathrm{cm}$. Now the transverse electric field pattern of the 15th mode in the uniform $25.4 \mathrm{~cm}$ waveguide could also meet the required boundary condition in a $2(12.7-1.7) \mathrm{cm}$ uniform waveguide, and in the region of the perturbation the average width of the guide is $2(12.7-1.62) \mathrm{cm}$. Hence, this could imply that of all the propagating modes in the uniform $25.4 \mathrm{~cm}$ waveguide the 15 th mode meets the boundary condition in the region of the perturbation most closely. Hence, the 15 th mode would be the most favored of all the higher modes in the region of the perturbation and $R_{15}$ the largest of all the mode conversion coefficients.

To support the above suggestion concerning the reason for the particular higher mode content observed, the experiment was repeated with another sinusoidal perturbation about $3 \lambda_{0}$ long and with $(h a)=3.05 \mathrm{~cm}$ instead of $2.54 \mathrm{~cm}$ as in the previous case. In this case the average height of the perturbation was $1 / 13$ the width of the unperturbed waveguide. The amplitude variations along the axis of the guide showed that the $\mathrm{TE}_{13.0}$ mode was the most predominant mode excited by the perturbation (fig. 10). The transverse amplitude variations plotted in figure 11 also very clearly show the interference of the $\mathrm{TE}_{13,0}$ mode.

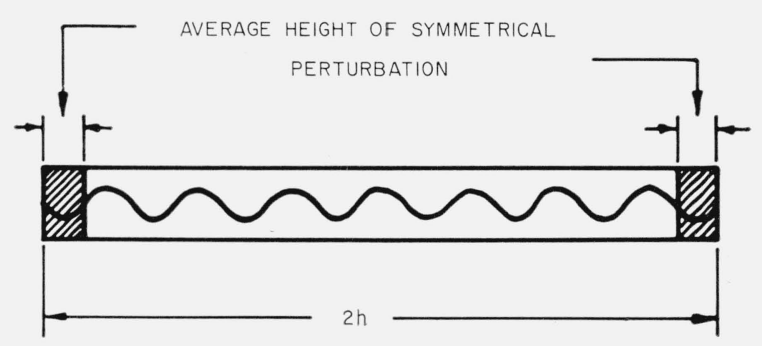

FIgure 9. Transverse variations of the electric field for the $T E_{0,15}$ mode showing situation when first null coincides with average height of perturbation. 


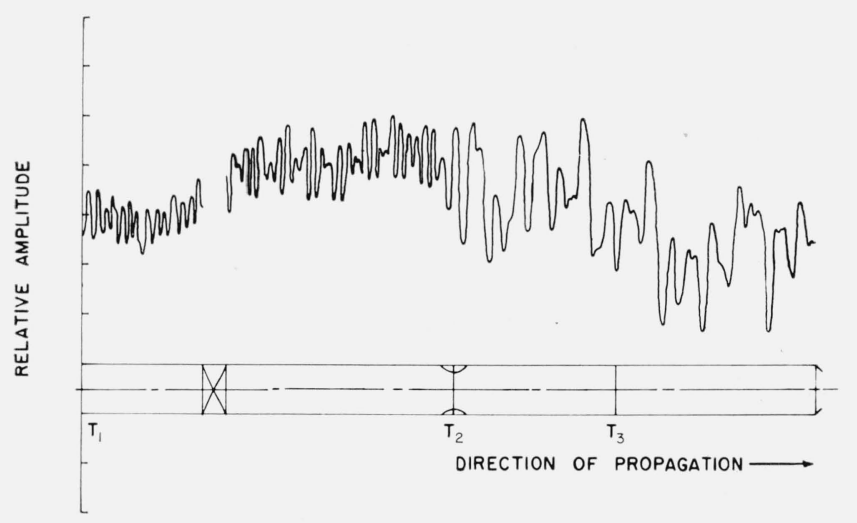

Figure 10. Axial amplitude variations with sinusoidal perturbation $\mathrm{a}=0.24$.

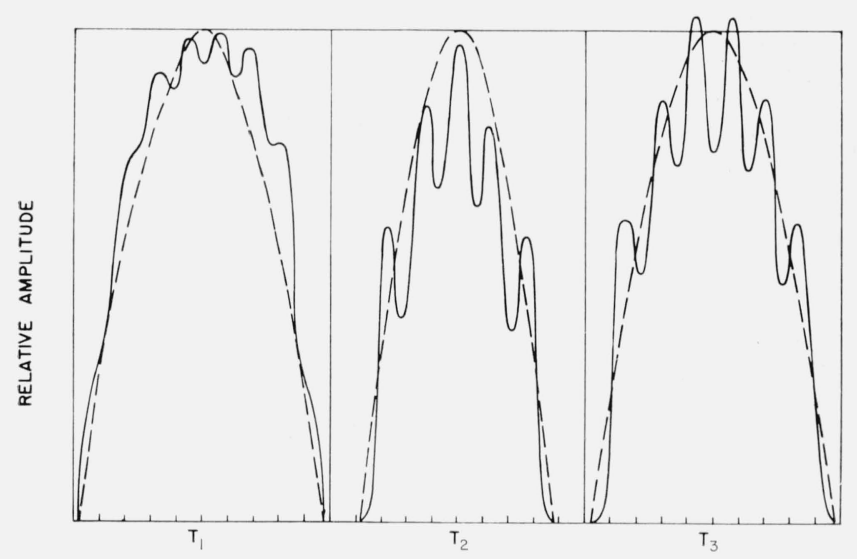

FIgURE 11. Transverse amplitude variations with sinusoidal perturbations $\mathrm{a}=0.24$.

Further experiments with sinusoidal perturbations showed that for perturbation lengths over $20 \lambda_{g 1}$, the mode conversion tends to become negligible if its height does not exceed 20 percent of the waveguide half width.

By narrowing a length of the waveguide to a uniform $20.3 \mathrm{~cm} \times 0.95 \mathrm{~cm}$ cross section, it was possible to simulate conditions similar to those encountered when the path of propagation is crossed by sunrise or sunset. In the case of a night to day path it was noted that more of the higher order modes were excited at the transition, being both reflected from it and transmitted beyond it. For the day to night path the lower order modes were the more predominant, but it was remarkable to note that in this case no matter what the shape of the transition joining the narrow waveguide to the broad one all the reflections from the transition region in the backward direction were negligible while the wave pattern beyond the transition region was rich in higher mode content as seen in figure 12 and figure 13 showing the longitudinal and transverse amplitude variations.

The above observations have suggested an approximate theoretical approach to the solution of prop-

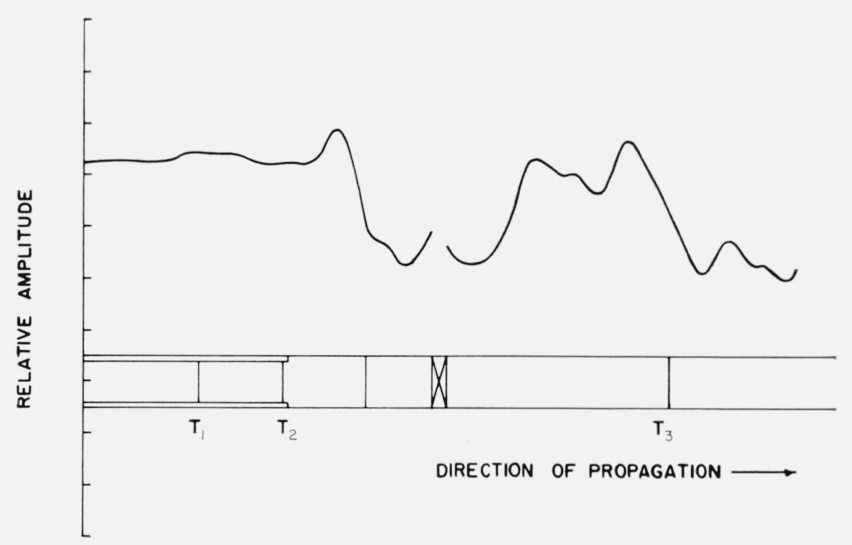

FIGURE 12. Axial amplitude variations for day to night transition path.

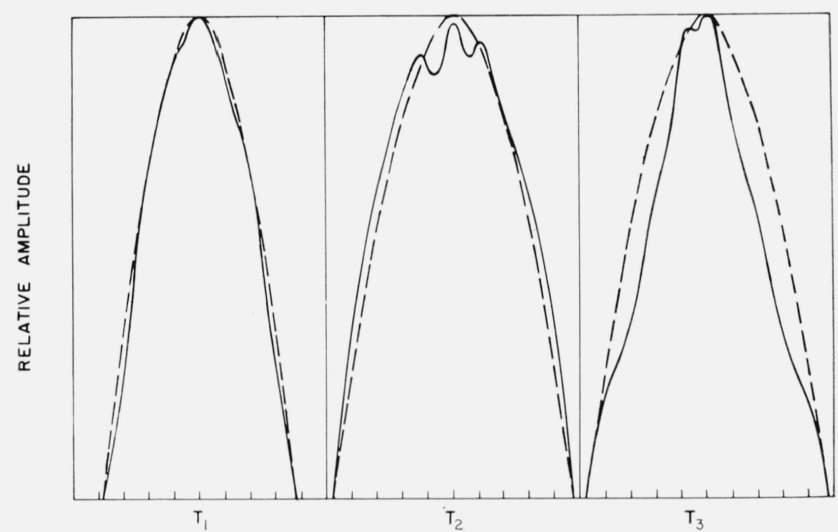

Figure 13. Transverse amplitude variations for day to night transition path.

agation problems in multimode waveguides yielding results that compare favorably with the experimental results. These solutions which will be dealt with in detail in a subsequent paper, predict also that the mode which is closest to being cutoff in the greater part of the transition region of the waveguide, tends to be predominant.

The mode closest to cutoff is that mode for which

$$
\cos \theta_{n}=\left[1-\left(\frac{n \lambda_{0}}{4 h(z)}\right)^{2}\right]^{1 / 2}
$$

is a minimum in the transition region. In the above equation $h=12.7 \mathrm{~cm}$ has been replaced by $h(z)$ the half width of the waveguide as a function of distance in the transition region. These theoretical results could also be applied to explaining the mode content in the cases when sinusoidal perturbations were introduced.

\section{Conclusions}

The experimental investigations thus far have shown that perturbations in the walls of multimode 
waveguides excite all orders of propagating modes traveling away from the perturbation. In all cases the predominant mode on both sides of the perturbation has been the lowest ordered mode. This is to be expected since energy incident upon the perturbation was predominantly in this mode. In most cases there was also a substantial amount of the $3 \mathrm{~d}$ mode (this would correspond to the second mode in the earth-ionosphere waveguide ${ }^{3}$ ); this was partly due to the fact that the incident wave contained some $3 \mathrm{~d}$ mode energy and partly because of conversion of energy from the lowest mode at the perturbation. The higher ordered modes observed were primarily caused by the presence of the perturbation. For the perturbations used it was found that the energy in modes 13 or 15 or both was substantially greater than that in modes 7,9 , and 11 . It was also observed that small changes in the width of the perturbation made a large change in the relative amplitudes of modes 13 and 15 . The general conclusion is that the distribution of energy among the various propagating modes is critically dependent upon the size of the perturbation. This is particularly true in the case of perturbations less than about three wavelengths long. For longer perturbations the mode conversion becomes less until at a length of 20 wavelengths (and a height less than a wavelength) there is hardly any noticeable mode conversion at all.

\footnotetext{
${ }_{3}^{3}$ The modes $1,3,5$, . . 15 in the model waveguide correspond to modes $1,2,3, \ldots 8$, respectively, in the earth ionosphere waveguide.
}

The measurement techniques have now been refined to the extent that quantitative comparisons can be made with theoretical solutions. It is expected that data on such comparison will become available soon.

\section{References}

Maley, S. W., and E. Bahar (1963), Model studies of the influence of ionosphere perturbations on VLF propagation, E.E. Dept. Univ. of Colo., Tech. Summary Rpt., Contract CST-7348, ARPA order No. 183-62.

Morgan, S. P., Jr. (1950), Mode conversion losses in transmission of circular electric waves through slightly noncylindrical guides, J. Appl. Phys. 21, 329-338.

Wait, J. R., and A. Murphy (1957), The geometric optics of VLF sky wave propagation, Proc. IRE 45, 754-760.

Wait, J. R. (1960a), Terrestrial propagation of VLF radio waves, J. Res. NBS 64D (Radio Prop.), No. 2, 153-204.

Wait, J. R. (1960b), A preliminary analysis of VLF mode propagation for a variable ionosphere height, (private communications) (see Wait, 1962).

Wait, J. R. (1962), Mode conversion in the earth-ionosphere waveguide, NBS Tech. Note 151.

Wait, J. R. (1964), Two-dimensional treatment of mode theory of the propagation on VLF radio waves, J. Res. NBS 68D (Radio Prop.), No. 1, 83.

\section{Additional Reference}

Wait, J. R. (1962), Analysis of VLF mode propagation for a variable ionosphere height, J. Res. NBS 66D (Radio Prop.), No. $4,453-461$.

(Paper 68D1-314) 